\title{
Infrared absorption from Charge Density Waves in magnetic manganites
}

\author{
P. Calvani, G. De Marzi, P. Dore, S. Lupi, P. Maselli, F. D'Amore, and S. Gagliardi \\ Istituto Nazionale di Fisica della Materia \\ and Dipartimento di Fisica, Università di Roma "La Sapienza", Piazzale A. Moro 2, I-00185 Roma, Italy \\ S-W. Cheong \\ ATEST Bell Laboratories, Murray Hill, New Jersey 07974, U.S.A. \\ and Department of Physics, Rutgers University, Piscataway, New Jersey 08855, U. S. A.
}

(May 10, 2018)

\begin{abstract}
The infrared absorption of charge density waves coupled to a magnetic background is first observed in two manganites $\mathrm{La}_{1-x} \mathrm{Ca}_{x} \mathrm{MnO}_{3}$ with $x=0.5$ and $x=0.67$. In both cases a BCS-like gap $2 \Delta(T)$, which for $x=0.5$ follows the hysteretic ferro-antiferromagnetic transition, fully opens at a finite $T_{0}<T_{\text {Neel }}$, with $2 \Delta\left(T_{0}\right) / k_{B} T_{c} \simeq 5$. These results may also explain the unusual coexistence of charge ordering and ferromagnetism in $\mathrm{La}_{0.5} \mathrm{Ca}_{0.5} \mathrm{MnO}_{3}$.
\end{abstract}

PACS numbers:75.50.Cc, 78.20.Ls, 78.30.-j

The close interplay between transport properties and magnetic ordering in the colossal magnetoresistance (CMR) manganites $\mathrm{La}(\mathrm{Nd})_{1-x} \mathrm{Ca}(\mathrm{Sr})_{x} \mathrm{MnO}_{3}$ is presently explained in terms of magnetic double exchange promoted by polaronic carriers along the path $\mathrm{Mn}^{+3}-\mathrm{O}^{-2}$ - $\mathrm{Mn}^{+4}$. [1] Charge hopping promotes the alignment of $\mathrm{Mn}^{+3}$ and $\mathrm{Mn}^{+4}$ magnetic moments, and vice versa. The polaronic effects are due to the dynamic Jahn-Teller distortion of the oxygen octahedra around the $\mathrm{Mn}^{+3}$ ions. The above mechanism explains how, in manganites with $0.2<x<0.48$, any increase in the magnetization enhances the dc conductivity, and vice versa. However, $\mathrm{La}_{0.5} \mathrm{Ca}_{0.5} \mathrm{MnO}_{3}$ shows an unpredicted coexistence of ferromagnetism and incommensurate charge ordering $(\mathrm{CO})$. This compound is paramagnetic at room temperature, becomes ferromagnetic (FM) at $T_{c} \simeq 225 \mathrm{~K}$ and, by further cooling (C), antiferromagnetic (AFM) at a Néel temperature $T_{N}^{C} \simeq 155 \mathrm{~K}$. [2] Upon heating the sample $(\mathrm{H})$ the FM-AFM transition is instead observed at $T_{N}^{H} \simeq 190$ $\mathrm{K}$. [3] The dc conductivity $\sigma(0)$ of $\mathrm{La}_{0.5} \mathrm{Ca}_{0.5} \mathrm{MnO}_{3}$ is quite insensitive to the PM-FM transition at $T_{c}$. [3] $\mathrm{X}$ ray, neutron [⿶] and electron diffraction [5] show quasicommensurate charge and orbital ordering in the AFM phase with wavevector $\vec{q}=(2 \pi / a)\left(\frac{1}{2}-\epsilon, 0,0\right)$. The incommensurability $\epsilon$ increases with temperature and follows the hysteretic behavior of the AFM-FM transition, until charge ordering disappears above the Curie point $T_{c}$. [5] At higher Ca doping, for $0.5 \lesssim x \lesssim 0.75$, a transition to a charge ordered phase [6] is observed in the paramagnetic phase at $T_{C O} . T_{C O}$ is a maximum $(265 \mathrm{~K})$ for $x=0.67 \simeq \frac{2}{3}$, where the charge ordering is commensurate with the lattice. Below $T_{C O}$, the system enters at $T_{N}$ an antiferromagnetic phase. For $x=0.67, T_{N} \simeq 140$ $\mathrm{K}$.

In the present paper, the unexpected coexistence of incommensurate charge ordering and ferromagnetism in $\mathrm{La}_{0.5} \mathrm{Ca}_{0.5} \mathrm{MnO}_{3}$ is investigated by infrared spec- troscopy. The spectra of stoichiometric $\mathrm{LaMnO}_{3}$ and of $\mathrm{La}_{0.33} \mathrm{Ca}_{0.67} \mathrm{MnO}_{3}$ are also examined for comparison. Both doped manganites allow to study the optical response of charge density waves (CDW) interacting with a magnetic background. Such an experiment could hardly be done in the low-dimensional systems where CDW are usually observed.

The present infrared spectra have been collected on the same $\mathrm{La}_{1-x} \mathrm{Ca}_{x} \mathrm{MnO}_{3}$ samples, prepared as described in Ref. [3], where the above diffraction studies [5.,6] have been performed. The oxygen stoichiometry has been accurately controlled, [5] to fulfill doping requirements which are particularly strict at $x=0.5$. The sintherized compound has been finely milled, diluted in CsI (1:100 in weight), and pressed into pellets under vacuum. One thus obtains reliable data when both the reflectance and the transmittance of the sample are too low, as shown in similar experiments [7] on $\mathrm{La}_{2-x} \mathrm{Sr}_{x} \mathrm{NiO}_{4}$ and $\mathrm{Sr}_{2-x} \mathrm{La}_{x} \mathrm{MnO}_{4}$. The infrared intensity $I_{s}$ transmitted by the pellet containing the oxide and that, $I_{C s I}$, transmitted by a pure CsI pellet, have been measured at the same $T$. One thus obtains a normalized optical density that, as shown in Ref. [8], is proportional to the optical conductivity $\sigma(\omega)$ of the pure perovskite, over the frequency range of interest here:

$$
O_{d}(\omega)=\ln \left[I_{C s I}(\omega) / I_{s}(\omega)\right] \propto \sigma(\omega)
$$

The spectra have been collected by two interferometers between 130 and $10000 \mathrm{~cm}^{-1}$, and by accurately thermoregulating the samples within $\pm 2 \mathrm{~K}$ between 300 and $20 \mathrm{~K}$. The susceptibility $\chi(T)$ of $\mathrm{La}_{0.5} \mathrm{Ca}_{0.5} \mathrm{MnO}_{3}$ has been measured at zero static field in a commercial apparatus by the Hartshorn method, at a frequency of 127 $\mathrm{Hz}$.

The optical density $O_{d}(\omega)$ of $\mathrm{La}_{0.5} \mathrm{Ca}_{0.5} \mathrm{MnO}_{3}$ is compared in Fig. 1 with that of stoichiometric $\mathrm{LaMnO}_{3}$. The latter compound shows in Fig. 1(a) a negligible absorption in the midinfrared at all temperatures. At least 10 
phonon lines, out of the 19 predicted [9] under the orthorombic symmetry of $\mathrm{LaMnO}_{3}$, are also found out in Fig. 1(a) by a fit to Lorentians. A detailed analysis of the phonon spectrum of this family of manganites will be reported elsewhere.

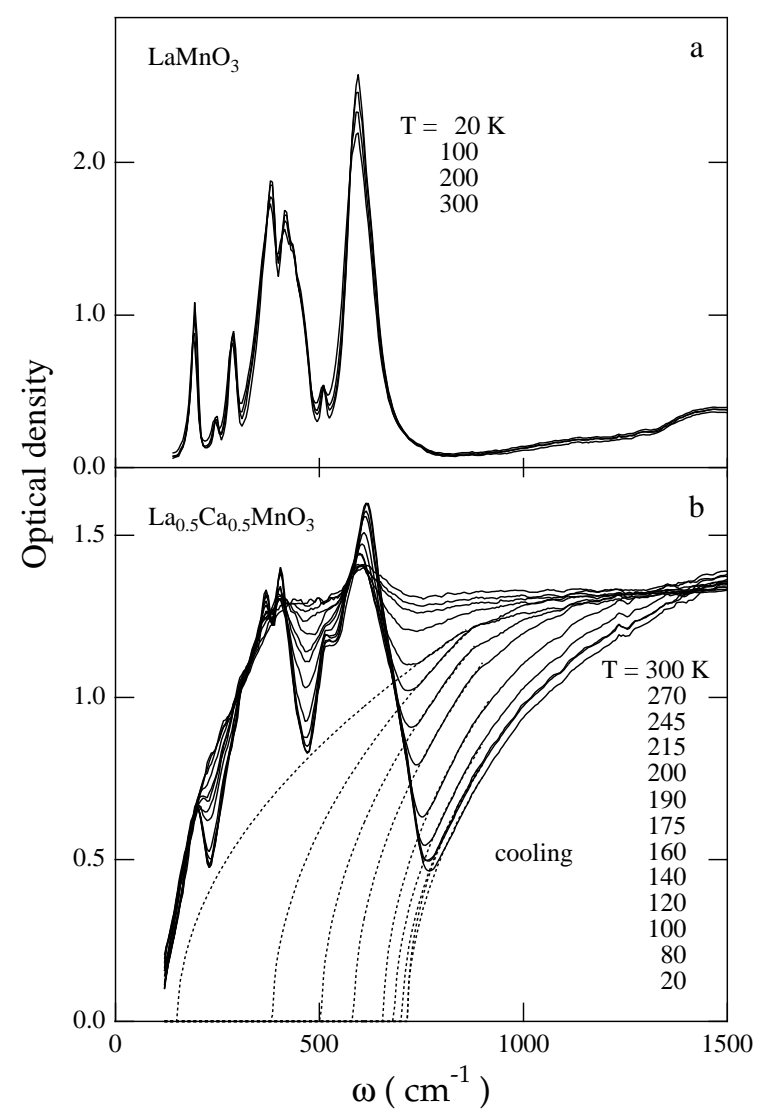

FIG. 1. The optical density $O_{d}$ of polycrystalline $\mathrm{LaMnO}_{3}$ (a) and $\mathrm{La}_{0.5} \mathrm{Ca}_{0.5} \mathrm{MnO}_{3}$ (b) as the sample is cooled from 300 $\mathrm{K}$ to $20 \mathrm{~K}$. The dashed lines in (b) represent extrapolations based on Eq. (3).

Let us now consider Fig. 1(b), where the optical density of $\mathrm{La}_{0.5} \mathrm{Ca}_{0.5} \mathrm{MnO}_{3}$ is reported for decreasing temperatures. At $300 \mathrm{~K}$ the phonon peaks are shielded by a broad background which, after a straightforward comparison with Fig. 1(a), can be attributed to the mobile 10 holes introduced by Ca doping. However, at a $T$ between 245 and $215 \mathrm{~K}$, a minimum appears in that background, which gradually deepens by further lowering the temperature. This signature of an optical gap provides evidence for increasing localisation of the carriers. Indeed, as $T$ lowers, phonon peaks similar to those in Fig. 1(a) become more and more evident, showing that the screening action of the mobile holes weakens. Moreover, the states lost in the gap region for decreasing temperature are transfered to higher frequencies (see the crossing point of the absorption curves at $\simeq 1500 \mathrm{~cm}^{-1}$ ), not to a coherent peak at $\omega=0$. This finding is consistent with the regular decrease in $\sigma(0)$ [3] observed in $\mathrm{La}_{0.5} \mathrm{Ca}_{0.5} \mathrm{MnO}_{3}$ between room temperature and $T_{N}$.

A loss of spectral weight such as in Fig. 1(b) can be measured by the variation of an effective number of carriers $n_{\text {eff }}(T)$, obtained by integrating $\sigma(\omega)$ between two suitable frequencies. By taking into account Eq. (1), we can define for the present purpose a quantity:

$$
n_{e f f}^{*}(T)=\int_{\omega_{1}}^{\omega_{2}} O_{d}(\omega) d \omega \propto n_{e f f}(T)
$$

As suitable integration limits one may take the two frequencies where $O_{d}(\omega)$ does not appreciably change with temperature, $\omega_{1}=200 \mathrm{~cm}^{-1}$ and $\omega_{2}=1500 \mathrm{~cm}^{-1}$. The values thus obtained for $n_{\text {eff }}^{*}(T)$ along a thermal cycle are plotted in Fig. 2(a). The inset compares the spectra observed at the same $T=160 \mathrm{~K}$ upon cooling and heating the sample. When the latter is cooled from $T \gtrsim T_{c}$, spectral weight is increasingly lost in the far infrared. A comparison with the corresponding curves of the magnetic susceptibility $\chi(T)$ in Fig. 2(b) shows that the decrease in $n_{\text {eff }}^{*}(T)$ is completed well below the Néel temperature $T_{N}^{C}$. Full localisation of the carriers is then observed only when $\chi(T)$ has reached its minimum value and an AFM phase is established in the whole sample. When heating the sample, $n_{\text {eff }}^{*}(T)$ starts increasing around $120 \mathrm{~K}$, again well below $T_{N}^{H}$.

The behavior of the infrared absorption in Fig. 1(b) and 2(a) in a temperature range where electron diffraction observes incommensurate charge ordering, points toward the formation a charge density wave. One can then extract from Fig. 1(b) the optical gap $2 \Delta(T)$ and compare its behavior with that expected for a CDW. In that case, $\sigma(\omega)$ at $\omega \gtrsim 2 \Delta(T)$ is similar to that of a semiconductor in the presence of direct band to band transitions. [11] By remembering again Eq. (1), one can then fit to the experimental $O_{d}(\omega)$, for $\omega \gtrsim 2 \Delta(T)$, the expression:

$$
\sigma(\omega) \propto[\omega-2 \Delta(T))]^{\alpha} .
$$

The curves thus obtained, reported as dashed lines in Fig. 1(b), well describe the resolved part of the gap profile at all temperatures with the same $\alpha=\frac{1}{2}$. The resulting values for $\Delta(T)$ are plotted in Fig. 2(c) as full circles, those corresponding to heating the sample as open circles. BCS-like fits for $2 \Delta(T)$ have been successfully applied to the optical behavior of charge density waves in polar systems, 12. even if the resulting values for $2 \Delta_{0} / k_{B} T_{c}$ are much higher than that (3.52) expected for a weak electron-lattice coupling. An analytic expression for $2 \Delta(T)$ between $T=0$ and $T_{c}$, 13 which also holds under moderately strong coupling, [14] can be written as:

$$
\Delta(T) / \Delta_{0}=\tanh \left[\frac{\Delta(T) T_{c}}{\Delta_{0} T}\right]
$$




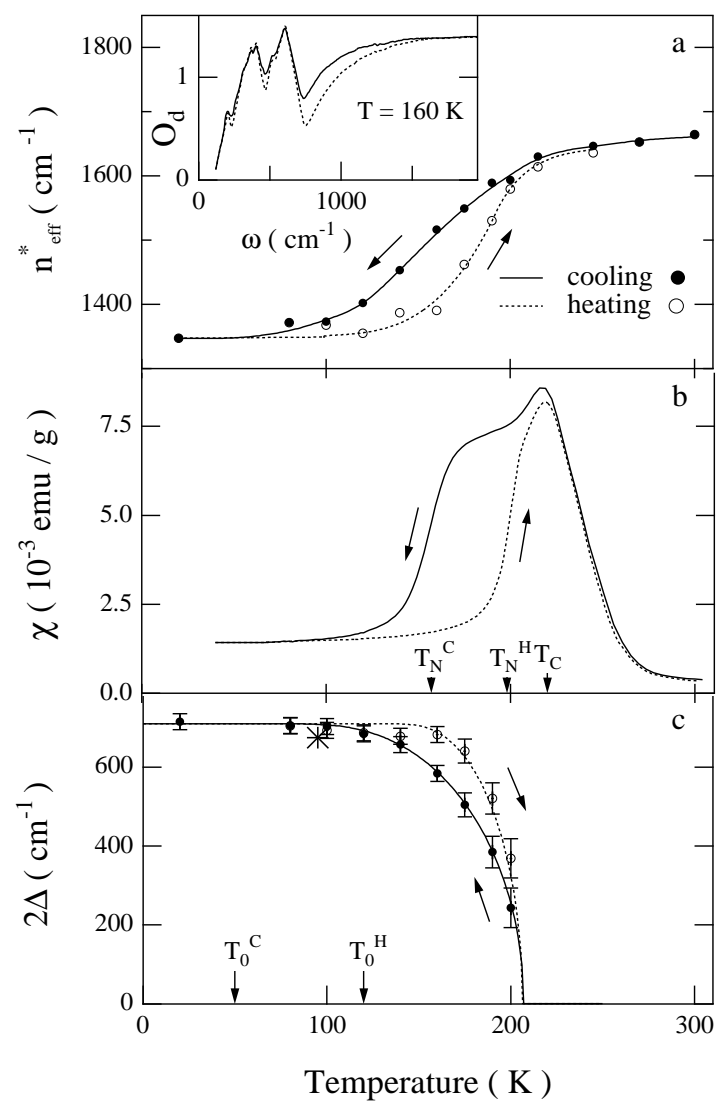

FIG. 2. Effective number of carriers $n_{\text {eff }}^{*}(T)$ (a), zero-field magnetic susceptibility $\chi(T)(\mathrm{b})$, and width of the optical gap $2 \Delta$ (c) vs. temperature in $\mathrm{La}_{0.5} \mathrm{Ca}_{0.5} \mathrm{MnO}_{3}$, as the sample is cooled (full circles, solid lines) or heated (open circles, dashed lines). The lines in (a) are guides to the eye, those in (c) are fits to Eq. (5). The inset in (a) shows the optical densities measured at the same $T=160 \mathrm{~K}$ by cooling (solid) and heating (dashed). In (b), the $T_{N}^{C}$ and $T_{N}^{H}$ values here found at a static field $H=0$ are slightly higher than reported in Ref. [3] for $H=1 \mathrm{~T}$. In (c), the star corresponds to the activation energy extracted from the dc resistivity of Ref. [3] (see text).

Unlike for an ordinary CDW, here the antiferromagnetic background fully localizes the charges at a finite $T_{0}<T_{N}$, which moreover depends on the thermal procedure. We find that this effects can be simply taken into account by replacing in Eq. (4) $T=0$ by $T_{0}^{X}(X=C, H)$ :

$$
\Delta(T) / \Delta_{0}=\tanh \left[\frac{\Delta(T)\left(T_{c}-T_{0}^{X}\right)}{\Delta_{0}\left(T-T_{0}^{X}\right)}\right]
$$

Indeed in Fig. 2(c), Eq. (5) well fits both series of data by using the same values $2 \Delta_{0}=710 \pm 20 \mathrm{~cm}^{-1}$ and $T_{c} \simeq$ $210 \mathrm{~K}$, which gives $2 \Delta_{0} / k_{B} T_{c} \simeq 4.8 . T_{0}^{C}=50 \mathrm{~K}$ and $T_{0}^{H}$ $=120 \mathrm{~K}$ are introduced into Eq. (5) for cooling and heating, respectively. Any attempt to raise either $T_{0}$ value towards the corresponding $T_{N}$ cosiderably worsen the fit, thus confirming that the gap fully opens well below the Néel temperature. One may notice that the dc resistivity reported for $\mathrm{La}_{0.5} \mathrm{Ca}_{0.5} \mathrm{MnO}_{3}$ in Ref. [3] follows an expo- nential behavior $\rho(T) \propto \exp \left[E_{0} / k_{B} T\right]$ below $\sim 110 \mathrm{~K}$. The activation energy $E_{0}=675 \pm 25 \mathrm{~cm}^{-1}$ (star in Fig. $2(\mathrm{c})$ ) is in excellent agreement with the present value of $2 \Delta_{0}$. A refinement of the present infrared data would instead be needed in order to investigate the nature of the gap (or possibly pseudogap) slightly above $T_{c}$.

The results of Fig. 2 can be usefully discussed in connection with those of electron diffraction in the same $\mathrm{La}_{0.5} \mathrm{Ca}_{0.5} \mathrm{MnO}_{3}$ powder. The behavior of the incommensurability parameter $\epsilon$, reported in Fig. 2 of Ref. [5], is quite similar to that of $n_{\text {eff }}^{*}(T)$ in Fig. 2(a). At $240 \mathrm{~K}$, where $n_{\text {eff }}^{*}(T)$ starts decreasing, weak peaks from a charged superlattice appear in the electron diffraction spectra, corresponding to $\epsilon \simeq 0.10$. As $T$ lowers, $\epsilon(T)$ decreases to 0.01 at $T=T_{N}$ and vanishes below $\sim 120$ $\mathrm{K}$. Along a thermal cycle, $\epsilon(T)$ follows a hysteretic behavior quite similar to that of $n_{\text {eff }}^{*}(T)$ in Fig. 2 (a). Such results, and the present observation of $2 \Delta(T)$, can be attributed either to an incommensurate, homogeneous charge density wave, or to discommensurations between ordered domains which are commensurate with the lattice. The former assumption can be ruled out at $x=0.5$ and in the presence of a strong electron-lattice coupling which creates polaronic carriers. The latter possibility points toward a phase separation scenario, as discussed below.

The average dimension of the commensurate domains would be [5] $L=a / p \epsilon$, where $a \simeq 0.55 \mathrm{~nm}$ is the lattice parameter in the Mn-O planes, and $p=2$ is the order of commensuration. This gives $L \simeq 5 a$ at $T_{c}, L \sim 50 a$ at $T_{N}$. One may expect that an AFM phase is established in such regions, as observed on a macroscopic scale below $T_{C O}$ for $x>0.5$. [6] If this is true, the ferromagnetism observed in $\mathrm{La}_{0.5} \mathrm{Ca}_{0.5} \mathrm{MnO}_{3}$ below $T_{c}$ can only take place in the disordered regions which separate the commensurate AFM clusters. Even there however, the charges should have a reduced mobility. To understand what may happen, one should consider that single-particle hopping undergoes intrinsical limitations at $x \geq 0.5$. Indeed, the dynamical distribution of $\mathrm{Mn}^{+3}$ and $\mathrm{Mn}^{+4}$ ions in the lattice is not purely statistical, as the system reduces its free energy by alternating distorted and undistorted octahedra. Then for $x=0.5$, a valence electron on $\mathrm{Mn}^{+3}$ is surrounded by $\mathrm{Mn}^{+4}$ nearest neighbors and may easily establish with these latters a double exchange mechanism. However, the same electron after the first jump will be surrounded by $\mathrm{Mn}^{+3}$ ions (except for the starting site, now $\mathrm{Mn}^{+4}$ ) so that a second jump in some forward direction is prevented by the Hubbard repulsion. Most electrons will then be confined within a few cells by jumping back and forth between Mn nearest neighbors, unless a coherent motion of charges over long distances takes place. However, this latter should determine a sudden drop in the dc resistivity below $T_{c}$, observed in 15 $\mathrm{Nd}_{0.5} \mathrm{Sr}_{0.5} \mathrm{MnO}_{3}$, not in 3] $\mathrm{La}_{0.5} \mathrm{Ca}_{0.5} \mathrm{MnO}_{3}$, where the small size of the $\mathrm{Ca}$ ion reduces the hybridization between 
the Mn $3 d$ and $\mathrm{O} 2 p$ orbitals. In $\mathrm{La}_{0.5} \mathrm{Ca}_{0.5} \mathrm{MnO}_{3}$, the hopping of polaronic carriers would then be able to establish a double-exchange mechanism, not effective enough to establish ferromagnetism and high dc conductivity in the whole sample.

The above phase-separation scenario for $\mathrm{La}_{0.5} \mathrm{Ca}_{0.5} \mathrm{MnO}_{3}$ at $T_{c}>T>T_{N}$ is consistent with the present spectra at infrared wavelengths, where any inhomogeneity on a scale much larger than $L$ is averaged out. In Fig. 1(b), the (poorly) mobile holes of the FM regions then appear as they were the excited states of the commensurate CDW condensate in the AFM clusters. The consistent behaviors of $\chi(T), n_{\text {eff }}(\mathrm{T})$, and $\epsilon(T)$ between $T_{c}$ and $T_{0}$ are easily explained: all those quantities in fact measure in different ways the increasing size of the AFM clusters at the expense of the FM regions.

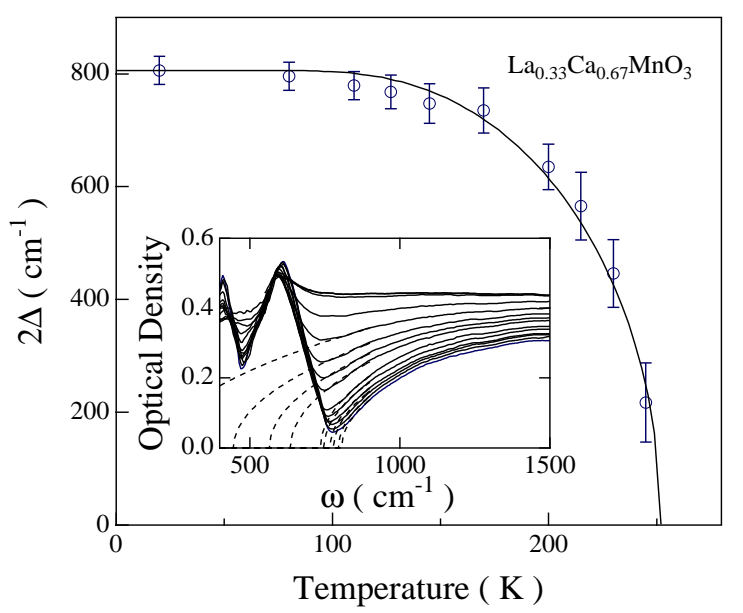

FIG. 3. Width of the optical gap $2 \Delta(T)$ in $\mathrm{La}_{0.33} \mathrm{Ca}_{0.67} \mathrm{MnO}_{3}$, with its fit to Eq. (5) (solid line). The spectra used to extract $2 \Delta(T)$ by the extrapolations based on Eq. (3) (dashed lines) are shown in the inset.

However, in order to firmly establish the above interpretation, it remains to show that the regions with commensurate $\mathrm{CO}$ may produce an infrared gap which behaves according to Eq. (5). To this purpose, we show in Fig. 3 the midinfrared spectra of $\mathrm{La}_{0.33} \mathrm{Ca}_{0.67} \mathrm{MnO}_{3}$. This sample exhibits commensurate charge ordering below $T_{C O}=265 \mathrm{~K}$, with wavevector $\vec{q}=(2 \pi / a)\left(\frac{1}{3}, 0,0\right)$. [6] Its absorption spectra, reported in the inset of Fig. 3, show formation of a gap in the infrared background, starting around $T_{C O}$. No appreciable effects around the PM-AFM transition at $T_{N} \simeq 140 \mathrm{~K}$, neither hysteretic phenomena, affect $2 \Delta(T)$, as obtained by use of Eq. (3). Also in the commensurate CDW of $x=0.67$, then, $2 \Delta(T)$ is well fit by Eq. (5). One obtains $T_{c} \simeq 255 \mathrm{~K}$ (in good agreement with the above $\left.T_{C O}\right), T_{0}^{C}=T_{0}^{H}=35 \mathrm{~K}$, and $2 \Delta_{0}=810 \pm 25 \mathrm{~cm}^{-1}$. One thus obtains $2 \Delta_{0} / k_{B} T_{c} \simeq$ 4.6, a value close to that observed in the FM phase of
$\mathrm{La}_{0.5} \mathrm{Ca}_{0.5} \mathrm{MnO}_{3}$.

In conclusion, the present paper first reports on the optical response of charge density waves interacting with a magnetic background. Both the incommensurate charge ordering in $\mathrm{La}_{0.5} \mathrm{Ca}_{0.5} \mathrm{MnO}_{3}$ and the commensurate ordering in $\mathrm{La}_{0.33} \mathrm{Ca}_{0.67} \mathrm{MnO}_{3}$ open in the infrared absorption a gap well described by a standard BCS-like equation, provided that $T=0$ is replaced with a finite $T_{0}<T_{N}$. The values for $2 \Delta_{0} / k_{B} T_{c}$ indicate a moderately strong coupling and authorize a posteriori the use of the above equation. These results also suggest an explanation for the intriguing coexistence of ferromagnetism and charge ordering in $\mathrm{La}_{0.5} \mathrm{Ca}_{0.5} \mathrm{MnO}_{3}$. It can be reconciled with the double-exchange model, provided that one introduces a phase-separation scenario. Commensurate charge-ordered clusters, probably antiferromagnetic, compete with disordered ferromagnetic domains where most charges are confined within a few cells. At the long infrared wavelengths, these poorly mobile holes appear as the excited states of the CDW condensate confined in the commensurate clusters.

\section{ACKNOWLEDGMENTS}

We wish to thank Denis Feinberg and Marco Grilli for many stimulating discussions.

[1] A. J. Millis et al., Phys. Rev. Lett. 74, 5144 (1995), and references therein.

[2] P. G. Radaelli et al., Phys. Rev. Lett. 75, 4448 (1995).

[3] P. Schiffer et al., Phys. Rev. Lett. 75, 3336 (1996).

[4] P. G. Radaelli et al., Phys. Rev.B 55, 3015 (1997).

[5] C. H. Chen and S-W. Cheong, Phys. Rev. Lett. 76, 4042 (1996).

[6] A. P. Ramirez et al., Phys. Rev. Lett. 76, 3188 (1996).

[7] P. Calvani et al., Phys. Rev. B 54, R9592 (1996).

[8] A. Paolone et al., Physica B 244, 33 (1998).

[9] M. Couzi and P. Van Huong, J. Chim. Phys. (France) 69, 1339 (1972).

[10] The non vanishing dc conductivity of $\mathrm{La}_{0.5} \mathrm{Ca}_{0.5} \mathrm{MnO}_{3}$ at $300 \mathrm{~K}\left(\sigma(0)=100 \Omega^{-1} \mathrm{~cm}^{-1}\right)$ 3]) does not contradict the absorption drop observed in Fig. 1(b) for $\omega<250$ $\mathrm{cm}^{-1}$. The latter effect is due to the penetration depth $\lambda \propto 1 / \sqrt{\sigma \omega}$ of the infrared radiation, which at low $\omega$ becomes comparable with the average dimension of the perovskite grains dissolved in CsI.

[11] P. A. Lee et al., Solid State Commun. 14, 703 (1974).

[12] T. Katsufuji et al., Phys. Rev. B 54, R14230 (1996).

[13] G. Burns, Solid State Physics, Academic Press, London 1985 , p. 649 .

[14] D. J. Thouless, Phys. Rev. 117, 1256 (1960).

[15] Y. Tokura et al., Phys. Rev. Lett. 76, 3184 (1995). 